This is the submitted version of the following article:

Solis-Tinoco V., Marquez S., Quesada-Lopez T., Villarroya F., Homs-Corbera A., Lechuga L. M.. Building of a flexible microfluidic plasmo-nanomechanical biosensor for live cell analysis. Sensors and Actuators, B: Chemical, (2019). 291. : 48 - . 10.1016/j.snb.2019.04.038,

which has been published in final form at https://dx.doi.org/10.1016/j.snb.2019.04.038 () https://dx.doi.org/10.1016/j.snb.2019.04.038. This manuscript version is made available under the CC-BY-NC-ND 4.0 license http: //creativecommons. org/licenses/by-nc-nd/4.0/ 


\title{
Building of a flexible microfluidic plasmo-nanomechanical biosensor for live cell analysis
}

\author{
V. Solis-Tinoco ${ }^{a}$, S. Marquez ${ }^{a}$, T. Quesada-Lopez ${ }^{b}$, F. Villarroya ${ }^{b}$, A. Homs-Corbera ${ }^{a}$ and L. M. Lechuga ${ }^{a^{*}}$
}

a. Nanobiosensors and Bioanalytical Applications Group (NanoB2A), Catalan Institute of Nanoscience and Nanotechnology (ICN2), CSIC, CIBER-BBN and BIST, Campus UAB, Bellaterra, 08193 Barcelona, Spain. E-mail: laura.lechuga@icn2.cat.

b. Department of Biochemistry and Molecular Biomedicine, Institut de Biomedicina (IBUB), University of Barcelona, Barcelona, Spain. CIBER Fisiopatología de la Obesidad y Nutrición, Madrid, Spain

tElectronic Supplementary Information (ESI)

Biosensor devices can constitute an advanced tool for monitoring and study complex dynamic biological processes, as for example cellular adhesion. Cellular adhesion is a multipart process with crucial implications in physiology (i.e. immune response, tissue nature, architecture maintenance, or behavior and expansion of tumor cells). This work focuses on offering a controlled methodology in order to fabricate a flexible plasmo-nanomechanical biosensor placed within a microfluidic channel as a new tool for future cell adhesion studies. We designed, fabricated, and optically and mechanically characterized this novel optical biosensor. As a proof-of-concept of its functionality, the biosensor was employed to observe fibroblasts adhesion in a cell culture. The device is configured by an hexagonal array of flexible rigid/soft polymeric nanopillars capped with plasmonic gold nanodisks integrated inside a microfluidic channel. The fabrication employs low-cost and large-scale replica molding techniques using two different polymers materials (EPOTECK OG142 and 310M). By using those materials the spring constant of the polymer nanopillars $(k)$ can be fabricated from $1.19 \mathrm{E}^{-02}[\mathrm{~N} / \mathrm{m}]$ to $5.35 \mathrm{E}^{+00}[\mathrm{~N} / \mathrm{m}]$ indicating different mechanical sensitivities to shear stress. Therefore, the biosensor has the feasibility to mimic soft and rigid tissues important for the description of cellular nanoscale behaviours. The biosensor exhibits a suitable bulk sensitivity of $164 \mathrm{~nm}$ or 206 $\mathrm{nm} /$ refractive index unit respectively, depending on the base material. The range of calculated forces goes from $\approx 1.98 \mathrm{nN}$ to $\approx .942 \mu \mathrm{N}$. This supports that the plasmo-nanomechanical biosensors could be employed as novel tool to study living cells behavior.

Therefore,

Keywords: nanofabrication, lab-on-a-chip, optical biosensor, tissue engineering, cell growth.

\section{Introduction}

Cell adhesion is an active process that cells undergo when they first interact with a substrate or with another cell. These interactions can last few seconds as in immunological signalling or can result enduring as in tissue formation. ${ }^{1}$ It is a crucial physiological event which impacts cellular behavior, tissue formation, architecture, immune response and tumor cell spreading. ${ }^{2}$ Although the nature of the static bonding process of a cell to a substrate is complex, numerous studies identify three main stages ${ }^{1,3,4}$ : cell attachment, cell body flattening, spreading, and conformation of focal adhesion sites between the cell and the substrate product of actin cytoskeleton reorganization. ${ }^{4}$ In other words, cells not only stick to the substrate, they form complex linkages between the extracellular matrix (ECM) and the intracellular actin cytoskeleton that provide a physical pathway to establish a bidirectional communication (integrin-ECM linkage). Failure in cell adherence will end in a compromised cell growth, proliferation, and even differentiation. Hence, it is paramount to develop controlled methodologies to create high-sensitive and non-invasive biosensing platforms capable of monitoring in real-time the cell attachment process.

The production of a biosensor with the previous characteristics is a technological challenge. Its design and fabrication requires the consideration of several aspects including the properties of the substrate, the nanostructuring of the sensor, the in-situ surface biofunctionalization to promote cell adhesion, and the noninvasive and real-time monitoring of the process. Regarding the properties of the substrate, the rigidity, the dimensions and topography of the ECM are important parameters that elucidate cell fate. Besides biochemical cues $^{5,6}$, cell phenotype and function can be regulated by biophysical cues of the substrate since the ECM presents a nanoscale topography and contains nanometer-sized proteins including collagen, fibronectin, and vitronectin. ${ }^{3}$ 
On the one hand, stiff substrates can stimulate cell adhesion 7 , promote cell spreading and proliferation ${ }^{8}$, facilitate cell differentiation ${ }^{9}$ and slow down the cell migration. ${ }^{10}$ On the other hand, topographical cues can impact on the cell shape, regulate cell proliferation and facilitate stem cell differentiation. ${ }^{11}$ For example, anisotropic arrays of polydimethylsiloxane (PDMS) micropillars promoted the elongation of epithelial cells along the axis of the stiffest direction, but this configuration reduced cell proliferation. ${ }^{12}$ Meanwhile, cylindrical arrays of PDMS micropillars showed no preferable alignment of epithelial cells. ${ }^{13}$ In another approach, differentiation of human Mesenchymal Stem Cells (hMSCs) was biased favouring osteogenesis on stiff arrays of PDMS micropillars with varying stiffness. ${ }^{14}$

Although several attempts have been made to study the interplay between the cell behaviour, the rigidity and topography of the substrate, the interwoven effects of both biophysical cues is still a field of research for tissue engineering and regenerative medicine. Besides the material rigidity and topography, biocompatibility plays also an important role for fabricating a substrate for cell culture. To enhance cell attachment, there are numerous biofunctionalization procedures for substrate preparation. ${ }^{6}$ In the case of nanopatterned surfaces, a common strategy consists of immersing the substrate in a fibronectin solution, letting fibronectin to be absorbed over the entire surface. ${ }^{15}$ In other approaches, proteins such as collagen and vitronectin are covalently linked by microprinting onto the substrate surface. ${ }^{16}$ Positively charged bioactive coatings can also be used to promote the initial attachment process by electrostatic interaction since the cellular membrane of some cells has a negative surface charge. ${ }^{17}$ Subsequently, cells are seeded into the pretreated surface by pipetting or using a polymerbased fluidic system. For instance, Yang et. al used a flexi-perm silicon chamber for cell culturing and a gravitybased perfusion system to control the surrounding solutions to the targeted cells. ${ }^{18}$ Although these processes are effective for substrate pretreatment, there is a lack of compatibility in the substrate dimensions and the volume of the solutions employed. It is not effective to integrate a nanopatterned surface within fluidic wells of macroscopic dimensions requiring solution in the order of milliliters.

In regard to the real-time monitoring tools, Localized Surface Plasmon Resonance (LSPR) is a highly sensitive and cost-effective optical technique for label-free biosensing, widely demonstrated in the literature, for example, for the detection of gluten peptides or tumor-associated autoantibodies. ${ }^{19,20}$ Owing to the high sensitivity of LSPR to local refractive index changes near the metal surface, the LSPR change can be used to study the cell behaviour and, thereby, to study the cell integrin-ECM linkage. On other hand, plasmonic nanostructures can be fabricated over polymer nanostructures to provide them height and flexibility. There are two approaches that have employed SPR-based sensors for studying cell adhesion, spreading and contractility. Wang et al. mapped a singlecell-substrate interaction by SPR microscopy when introducing osmotic pressure changes into the cell environment. Yang et al. proposed an alternative approach named Long Range Surface Plasmon Resonance (LRSPR) to monitor the vertical displacement of the cell membrane of 3T3 fibroblasts and cancer cells associated with the cell micromotion mechanism. ${ }^{21}$ While these approaches are suitable for studying cell behaviour, fabricating a clever biosensor combining both mechanical and optical properties may provide an efficient and low-cost detection platform capable of monitoring the cell-binding process when studying the interwoven effects of stiffness and topography of the nanosubstrate.

In our approach, we developed a plasmo-nanomechanical biosensor for detecting the adhesion and spreading of live fibroblasts both by changes of the refractive index in the environment as well as interactions between gold plasmonic nanodisks placed on flexible polymer nanopillars. The biosensor consists of a hexagonal array of closely spaced, vertical, elastomeric nanopillars capped with gold nanodisks embedded in a microfluidic channel. The fabrication of the biosensor is based on a replica molding technique that we reported previously. ${ }^{22,23}$ The elastomeric nanopillars are designed to mimic soft or rigid tissues using two new polymer materials (EPOTECK OG142 and 310M). The biosensor combines the mechanical flexibility of polymer nanopillars with the optical properties of plasmonic gold nanodisks that exhibit a LSPR response. LSPR is advantageous for studying cell adhesion and spreading owing to its low penetration depth (30-50 nm) compared to other SPR configurations (SPR 100-200 nm, and LRSPR 500-1000 nm). ${ }^{21}$ Finally, the optical detection does not require significant computational processing.

Herein, we underpin the following contributions: i) polymer nanopillars can support gold plasmonic nanodisks to detect not only changes in the refractive index of the medium but also the deflection of the flexible nanopillars by a shear force, ii) the nanopillar deflection is not limited to only one direction, iii) the spring constant of the 
nanopillars can be tuned changing their geometric dimensions and structural material to mimic the rigidity of different tissues, iv) the array of polymer nanopillars can be fabricated using biocompatible materials facilitating their integration within a microfluidic channel for reducing the biosensor dimensions and ensuring at the same time the use of small volumes of reagents, and v) by using an integrated microfluidic system, the biosensor can be easily functionalized to promote cell adhesion, if required. Our work faces the technological challenge of reproducing an adequate environment for cell interaction in an aim to mechanically imitate a physiological matrix resembling differential tissues in the human body. This, in order to study the existing cellular interactions at nanoscale while optimizing the available technological resources.

\section{Materials and methods}

\section{Fabrication of the biosensor}

The plasmo-nanomechanical biosensor design consists of an hexagonal array of closely spaced, vertical, elastomeric nanopillars capped with plasmonic gold nanodisks embedded in a microfluidic channel. The fabrication of the biosensor includes the following steps: 1) fabrication of a Si master mold, 2) production of a patterned PDMS stamp from the master mold, 3) generation of a polymer replica of the original template, 4) evaporation of plasmonic gold nanodisks on the polymer nanopillars by e-beam evaporation 22,23 and 5) bonding of the biosensor, as shown in Fig. 1.

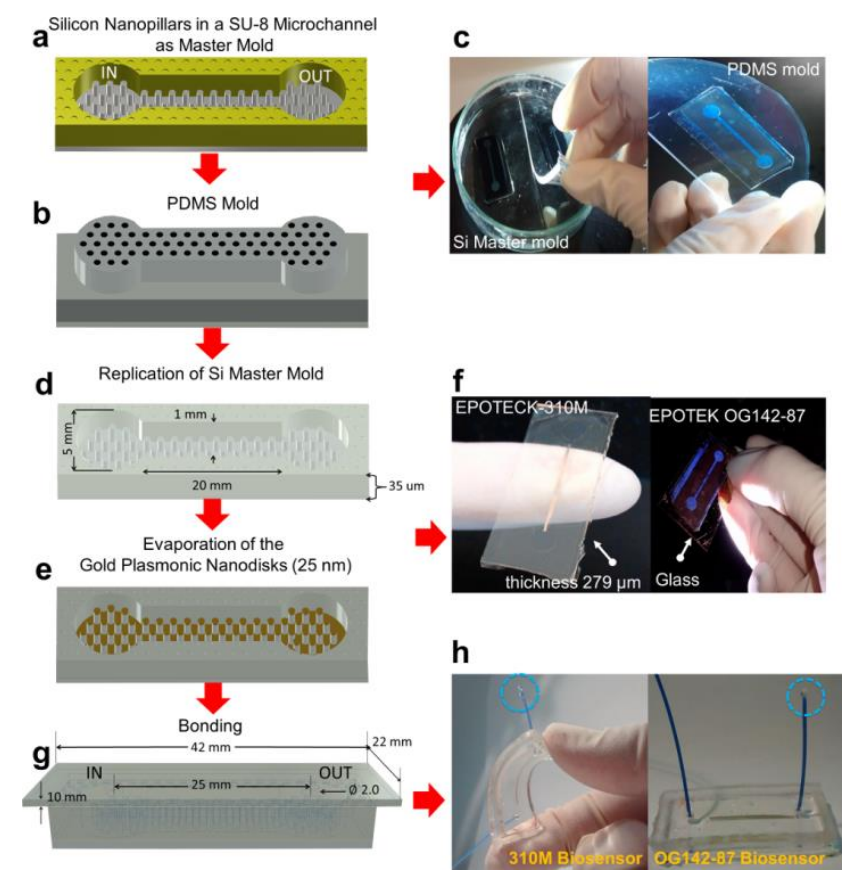

Fig. 1 Scheme of the fabrication process of the plasmo-nanomechanical biosensor. (a) Formation of Si nanopillars in the SU-8 microfluidic channel by catalytic etching. (b) PDMS mold of nanoholes with depths equal to the heights of the SiNPs. (c) Pictures showing a PDMS mold. (d) Polymer replica of the silicon master mold. (e) Gold nanodisks deposited onto the polymer nanopillars inside the microchannel. (f) Photo of a thin metallized polymer replica over a PDMS film and a glass substrate, respectively. (g) Bonding of the biosensor showing its real dimensions. (h) Photos of rigid and soft mimicking biosensors with water flowing in the microfluidic channel. 
The master mold consists of a SU-8 microfluidic channel patterned onto a silicon wafer that contains silicon nanopillars (SiNPs) inside this domain, as Fig. 1a shows. The fabrication process includes three main steps: (1) fabrication of the SU-8 microfluidic channel using photolithography, (2) preparation of the catalytic Au layer inside the channel, and (3) fabrication of SiNPs by metal-assisted-chemical etching of silicon according to our previous work. ${ }^{22}$ The silicon master mold is designed to have arrays of SiNPs with diameter $\left(d_{r}\right)$ of $167.5 \pm 11 \mathrm{~nm}$ and height $(h)$ of $294 \pm 46 \mathrm{~nm}$ with a center-to-center distance $\left(d_{c c}\right)$ of $400 \mathrm{~nm}$ inside the SU-8 microchannel (Fig. 1a). ${ }^{23}$

\section{PDMS mold}

The methodology described in ${ }^{23}$ was used to create a $1 \mathrm{~mm}$ thickness polydimethylsiloxane (PDMS) replica of the silicon master mold (Fig. 1b). Briefly, the surface of the silicon master was cleaned and oxidized by an oxygen plasma cleaner (Femto plasma cleaner, Electronic Diener Inc, Germany) at $75 \mathrm{~W}$, with an oxygen flow rate of $15 \%$ for $2 \mathrm{~min}$. Then, the surface of the master mold was treated with an aliquot (50 $\mu \mathrm{l})$ of trichlorosilane (tridecafluoro-1,2,2-tetrahydrooctyl) in a vacuum desiccator for $2 \mathrm{~h}$ at room temperature to deposit a monolayer onto the silicon nanopillars. This monolayer helps during the unmolding step by avoiding permanent sticking of the polymers to the master mold. As follows, the PDMS prepolymer and curing agent (DOW Sylgard 184) were thoroughly mixed in a volume ratio of 10:1, followed by a degassing step of 20 min to remove air bubbles. The degassed PDMS mixture was gently poured onto the silicon master and a second degassing step was applied for $1 \mathrm{~h}$. The PDMS was cured at $100^{\circ} \mathrm{C}$ for $1 \mathrm{~h}$ on a hot-plate (KW-AH, Chemat Technology Inc, United States). Finally, the cured PDMS was carefully unmolded from the Si master, as shown in Fig. 1c.

\section{Replication of the Si master mold and production of gold nanodisks}

The UV curable polymer EPO-TEK OG142-87 and thermal EPO-TEK 310M were used to produce the replica of the silicon nanopillar arrays integrated in the SU-8 microchannel. The process was based on a methodology described $i^{23}$. For the UV curable polymer, $1 \mathrm{~g}$ of polymer was poured on the PDMS and placed in a vacuum desiccator in order to remove air bubbles and fill the nanoholes. The mold was then covered with a precleaned glass slide or a PDMS film. Next, the polymer was exposed to UV light ( $\approx 190 \mathrm{~mW}$ at $365 \mathrm{~nm}, 4 \mathrm{~min})$ for $2 \mathrm{~h}$ at $150^{\circ} \mathrm{C}$. For the thermal polymer, $1 \mathrm{~g}$ of polymer with a mixing ratio of 10:5.5 was poured onto the PDMS. The same steps as the UV curable polymer were followed except for the curing process. The thermal polymer was cured in two subsequent steps: 1 day at room temperature and $2 \mathrm{~h}$ at $65^{\circ} \mathrm{C}$ on a hot-plate. After curing, the polymer structures were carefully peeled off from the PDMS mold (Fig. 1.d). Finally, a gold film of $\approx 25 \mathrm{~nm}$ thickness was deposited by e-beam evaporation on the polymer nanopillars forming gold nanodisks (Fig. 1.e). Fig. 1.f shows a polymer replica with the gold nanodisks (thickness $279 \pm 25 \mu \mathrm{m}$ ) using a PDMS film and a glass slide as base support, respectively.

\section{Microfluidic header}

The microfluidic header of the biosensor was created by enclosing the polymer microfluidic channel with integrated gold-capped nanopillars using a PDMS slab as lid, see Fig. 1g. PDMS has been widely used for fabricating chip-based microfluidic devices due to its ease of fabrication, low-cost, biocompatibility, elastomeric properties, and optical properties. Although a number of microfluidic devices employ oxygen plasma as the main bonding technique due to its excellent bonding results 24,25 , herein we used a non-permanent strategy to bond materials of different composition. For our particular application, a non-permanent bond of materials was required for seeding the cells without any leaking, but also for removing the PDMS lid for SEM analysis of adherent cells over the sensors. The bonding strategy combines oxygen plasma to clean the surfaces and a partial curing of PDMS (with a mixing ratio of 25:1) to bond a PDMS lid to the polymer (310M, OG142-87) replicas (see supporting information †). Once the bonding was done, two PEEK tubes (Valco Instruments Co. Inc. JR-T-6009) of $250 \mu \mathrm{m}$ of i.d. were affixed to each reservoir, see Fig. 1h. 

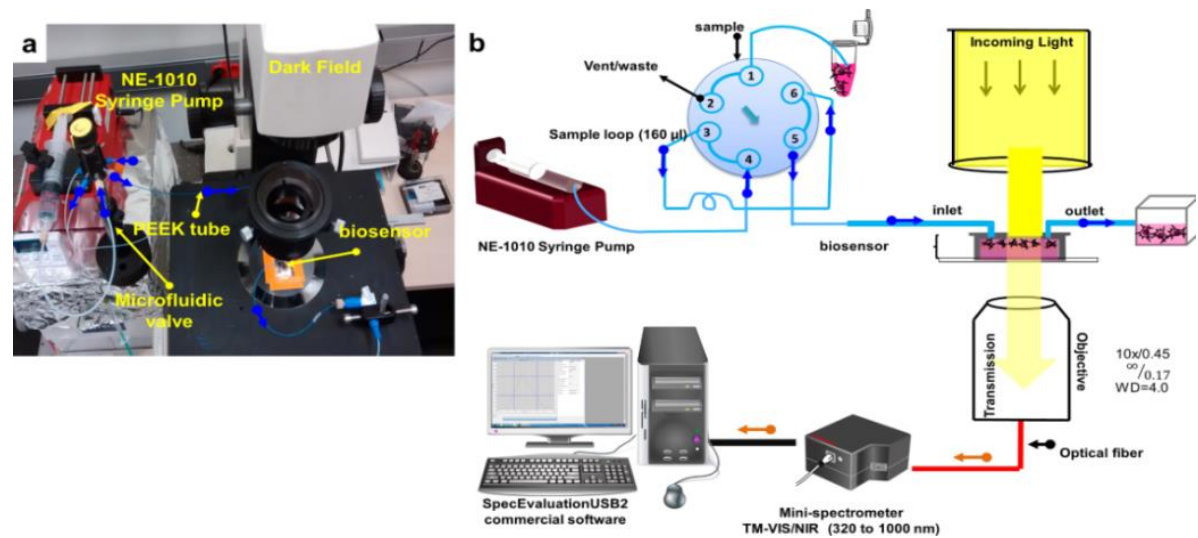

Fig. 2 Configuration of the DF microscope for transmission measurements. (a) Top-view photograph of the experimental optical setup with the microfluidic system. (b) Schematic showing the configuration for transmission evaluation of the plasmo-nanomechanical biosensors.

\section{Experimental set-up}

Fig. 2 shows a photo and a schematic drawing of the experimental setup employed for the biosensor characterization using a transmission configuration. The spectra were recorded with an inverted Nikon inspection microscope (Eclipse Ti-U Dark-Field (DF), Fig. 2.a), equipped with a halogen light source (100 W) and fiber-coupled $(\varnothing \mathrm{F}=1000 \mu \mathrm{m})$ to a mini-spectrometer (TM-VIS/NIR, Hamamatsu (320 to $1000 \mathrm{~nm})$ ). The biosensor was placed under the excitation light source (effective captured area with a diameter of $\left.100 \mu \mathrm{m}\left(7500 \mu \mathrm{m}^{2}\right)\right)$. A microfluidic syringe pump with adjustable pumping speed was used to assure a constant flow rate of reagents inside the microfluidic channel. Delivery of samples was done manually using an injection valve, see Fig. $2 b$. The measurements were performed by recording the LSPR spectra through an optical fiber (1000 $\mu \mathrm{m})$ from the DF microscopy (objective 10x) to the spectrometer.

For data acquisition, the commercial software, SpecEvaluationUSB2, supplied with the mini-spectrometer was employed. Normalized transmission spectra $(I s)$ were obtained by first subtracting a previously stored dark spectrum ( $d s$ ) from the data, and then dividing the result by a previously stored reference spectrum (Rs). The acquisition times for the spectra were ranged from $2 \mathrm{~s}$ to $4 \mathrm{~s}$ with 500 spectral accumulations. The spectra were saved and analyzed using Matlab and OriginLab software to calculate the spectral LSPR dip position ( $\lambda$ LSPR). A home-made script in Matlab fitted the transmission spectra with a high-degree polynomial.

\section{Bulk sensitivity evaluation}

To examine the bulk sensitivity of the biosensor to refractive index changes, we performed transmission measurements at normal incidence, as shown in Fig. 2. Moreover, given the complex configuration of our plasmonanomechanical biosensor, Finite-Difference Time-Domain (FDTD) simulations were carried out to study the transmission and electromagnetic field distribution at normal incidence of light in air and water (see supplementary supporting information ${ }^{\dagger}$ ).

Then, two different biosensors with different aspect ratio and spring constants were evaluated to find out the effect of their characteristics on the device sensitivity. The biosensors were the 310M biosensor $\left(d_{\text {pnp }}=160\right.$ $\mathrm{nm}, \mathrm{h}=320 \mathrm{~nm}$ ) with a spring constant of $1.19 \mathrm{E}^{-02}[\mathrm{~N} / \mathrm{m}]$ and the $0 \mathrm{G} 142$ biosensor $\left(\mathrm{d}_{\mathrm{pnp}}=185 \mathrm{~nm}, \mathrm{~h}=490 \mathrm{~nm}\right)$ with a spring constant of $5.35 \mathrm{E}^{+00}[\mathrm{~N} / \mathrm{m}]$. Four different concentrations of water/glycerol were prepared and the change in the refractive index contrast $(\Delta \mathrm{RI})$ were measured by a refractometer (ABBE model $315, \mathrm{UK})$. The samples employed were $2.5 \%(\Delta n \approx 0.0032), 5 \%(\Delta n \approx 0.0064), 7.5 \%(\Delta n \approx 0.0096)$ and $10 \%(\Delta n \approx 0.0128)$ of glycerol. The serially diluted glycerol solutions with known RI were injected into the microchannel at $10 \mu \mathrm{L} \cdot \mathrm{min}^{-1}$ and the induced $\Delta \lambda_{\text {LSPR }}$ was acquired. MilliQ water $(n \approx 1.3331)$ was employed as a continuous running solution. Finally, 
the bulk sensitivity $\left(S_{B}\right)$ for each biosensor was extracted from the slope of the linear fitting of the LSPR shift and the refractive index change $\left(\Delta \lambda_{\mathrm{LSPR}}\right.$ vs RI).

\section{Feasibility of the biosensors for fibroblasts adhesion}

The designed biosensors comprise a complex surface with a nanopatterned topography, stiffness and new composite materials. For this reason, it was important to verify the biocompatibility of the device with cell behaviour, specifically cell adhesion. To test this, a fibroblasts culture was performed. Pre-pleated fibroblasts were trypsinized (Gibco, 25300-054) and left to attach to the biosensors for 3 hours ${ }^{26}$ (for detailed fibroblasts culture protocols, refer to supplementary supporting information ${ }^{\dagger}$ ). Briefly, to avoid the biosensor damage with sterilizing protocols, UV light exposure was employed. The biosensors were exposed to the hood UV light for 10 minutes. Subsequently, they were immersed in a poly-L-lysine (P2636, Sigma) solution at a final concentration of $0.02 \mathrm{mg} / \mathrm{mL}$ and incubated for another 20 minutes. Poly-L-lysine was aspirated (vacuum, Figs. S2a-c ${ }^{\dagger}$ ) and washed with phosphate saline buffer (PBS). PBS was subsequently aspirated and the biosensors were left to dry under the hood for another 20 minutes. Pre-plated fibroblasts were trypsinized and counted with an automated cell counter (Eppendorf, Centrifuge 5702), see Fig. S2d †. Fibroblasts were plated at a density of $30 \times 10^{4}$ cells $/ \mathrm{mL}$ on the biosensors and on the conventionally employed dishes (100 mm x $20 \mathrm{~mm}$, Corning 15430167) as a control, see Fig. S2 ${ }^{\dagger}$. Fibroblasts were left to adhere to the surfaces for three hours in the presence of basal media at 37 ${ }^{\circ} \mathrm{C}$ with $7 \% \mathrm{CO}_{2}$ in the incubator, see Fig. S2f†. Monitoring of samples was carried out every 30 minutes using an optical microscope and acquired images at 20 amplifications, see Fig. $\mathbf{S 2 g}{ }^{\dagger}$. Finally, after $3 \mathrm{~h}$ of incubation, the samples were washed and the adhered fibroblasts were fixed ${ }^{27}$ for obtaining scanning electron microscopy (SEM) images for analyses, see Fig. S2ht.

\section{Effect of the culture media on the LSPR response}

The basal media employed for fibroblasts culture contains Dulbecco's Modified Eagle Medium (DMEM, Biowest L0104-500). This DMEM is supplemented with red phenol as a pH indicator and several other components. For this reason, two subsequent tests were performed in order to discard media components interference on the LSPR measurements. Following cell culture protocols and employing the $310 \mathrm{M}$ biosensor (see supplementary supporting information ${ }^{\dagger}$ ). First, we tested $\mathrm{pH}$ variance during the quantification timings. For this, the basal media alone (DMEM) was monitored every 30 minutes for three hours. Second, we assessed if cell respiration (consumed oxygen and produced carbon dioxide) could affect the LSPR spectra measurements. For this experiment, fibroblasts were incubated at the previously mentioned density $\left(30 \times 10^{4} \mathrm{cell} / \mathrm{mL}\right)$ in basal media for 3 hours and subsequently centrifuged ( $1.2 \mathrm{rpm}$ for 4 minutes). The obtained supernatant without fibroblasts was delivered into the microfluidic channel of the biosensor and the transmission spectra was recorded.

\section{LSPR response of fibroblasts}

As a proof-of-concept, we measured the transmission spectra for $3.0 \mathrm{~h}$ during fibroblast seeding on the different biosensors. The experiment goal was to demonstrate the plasmo-mechanic principle of operation of the biosensors by studying the nanopillar spring constant effect on cellular adhesion. For this, two $310 \mathrm{M}\left(\mathrm{d}_{\mathrm{pnp}}=160\right.$ $\mathrm{nm}, \mathrm{h}=320 \mathrm{~nm})$ and two OG142 $\left(\mathrm{d}_{\mathrm{pnp}}=185 \mathrm{~nm}, \mathrm{~h}=490 \mathrm{~nm}\right)$ biosensors were used with a spring constant of 1.19E$02[\mathrm{~N} / \mathrm{m}]$ and $5.35 \mathrm{E}^{+00}[\mathrm{~N} / \mathrm{m}]$, respectively. By following the seeding protocol (see supporting information $\mathrm{t}$ ) $30 \times 10^{4}$ cells $/ \mathrm{mL}$ were passed through the microchannel for 25 minutes. Then the outlet of the biosensor was closed in order to avoid the loss of the sample, as shown in Fig $2 \mathrm{~b}$. The fibroblasts were observed by DF microscopy to verify their survival and availability in the inspection zone. LSPR transmission spectra were obtained every 30 minutes for 3 hours.

\section{Results and Discussions}




\section{Characterization of the plasmo-nanomechanical biosensor}

PDMS molds of nanoholes were fabricated from silicon masters molds according to the controlled procedure described in ${ }^{23}$. Fig. 3.a shows a SEM image of a PDMS mold with a surface where a gold layer was deposited. The PDMS pattern (thickness $1 \mathrm{~mm}$ ) contains the hexagonal array of nanoholes whose depths correspond to the heights of the SiNPs. Differences observed in the diameter of replicated nanopillars, and the presence of defects in the replicated mold can be explained by fabrication tolerances in the master molds. The use of PDMS molds is inexpensive and multiple copies can be replicated using the original master mold (around 25). These features make PDMS molds an attractive, effective and low-cost methodology for fabricating sensors.

Two different arrays of polymer nanopillars (310M and OG142-87) were replicated from the same silicon master mold as shown in Fig. 3.b and c, respectively. It is important to highlight that the mechanical properties of these new materials (e.g., viscosity and Young's Modulus) are excellent to fully fill in the PDMS mold, unmold and keep vertically aligned nanopillars. Table S1 t at supporting information compares the experimental aspect ratio of the polymer nanopillars capped with gold nanodisks (thickness $=25 \mathrm{~nm}$ ) with their corresponding values in the silicon master mold. The results show an increase in the diameters of the OG142-87 nanopillars $\left(d_{\text {pnp }}\right)$, and also, an increase in the heights of both types. As a result, the aspect ratios of the polymer nanopillars showed to be greater than those of Si nanopillars masters. An explanation for this could be the temperature at which the different polymer nanostructures were exposed during the formation of the gold nanodisks by e-beam evaporation, causing these distortions. These results were consistent with $23,28,29$.
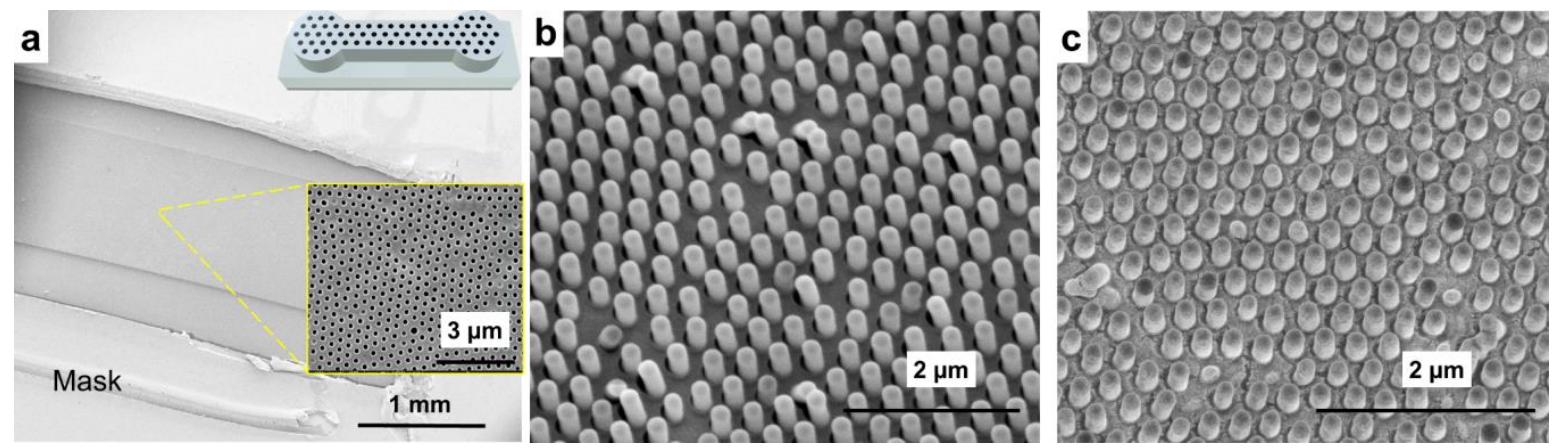

Fig. 3 (a) PDMS mold with a mesh of nanoholes within a microchannel (b) Epotek-310M nanopillars, and (c) EpotekOG142-87 nanopillars ( $15^{\circ}$ tilt view).

Other distortion might also be produced during the unmolding step since some polymer nanopillars adopted the orientation at which they were peeled-off from the PDMS mold. Table S1 $†$ shows the spring constant $(k)$ that the polymer nanopillars display according to their aspect ratio and the Young's Modulus of the material. $k$ not only represents the mechanical sensitivity (stiffness) of the biosensors to a shear stress, but also the mimicked soft or rigid tissues. The calculation of the $\mathrm{k}$ was extracted from the formula 1.1.14,30-33 For a cylinder of radius $r$ and length $L$, the resulted deflection caused by a force $F$ is defined as:

$$
\mathrm{F}=\mathrm{k} \cdot \Delta \mathrm{x}=\left(\frac{3}{4} \pi \mathrm{E} \frac{\mathrm{r}^{4}}{\mathrm{~L}^{3}}\right) \Delta \mathrm{x}
$$

where $E \mathrm{E}, k$, and $\Delta x$ are the Young modulus, the spring constant and the deflection of the pillar, respectively. To identify the corresponding mimicked tissue of each biosensor configuration, the aspect ratio and the Young Modulus were substituted in $1.1 \mathrm{k}=\left(\frac{3}{4} \pi \mathrm{E} \frac{\mathrm{r}^{4}}{\mathrm{~L}^{3}}\right)$, and then the results were compared with experimental data of 
the elastic moduli of mammalian tissues. As a result, SiNPs replicated using 310M and OG142-87 polymers can mimic the mechanical properties of tissues such as muscle and bone, respectively.

Fig. 1.h shows photos of the flexible and rigid biosensor devices. According to the dimensions of the microchannel (see Figs. 1.d and g) the Reynolds number is 142.2, which corresponds to a laminar flow regime. For preventing water leaking during the injection of samples through the microchannel, we tested the nonpermanent bond of materials. The experiment consisted of injecting water into OG142 and 310 sensors at different flowrates during $4 \mathrm{~h}$. The flow rate was ranged from $5 \mu \mathrm{L} / \mathrm{min}$ to $90 \mu \mathrm{L} / \mathrm{min}$. When the flow rate reached $90 \mu \mathrm{L} / \mathrm{min}$ ( $P_{\text {exerted over the walls }} \approx 0.01285 \mathrm{~Pa}$ ), the bond failed for both sensors. Thus, in our experiments, sample injection was done at a flow rate of $10 \mu \mathrm{L} / \mathrm{min}$.

\section{Evaluation of the bulk sensitivity}

As mentioned above, the bulk sensitivity of the biosensors was evaluated. Figs. $4 a-b$ show the time-resolved response of the biosensors. As expected, all sensograms showed a stepwise increase in the $\lambda$ LSPR as the surrounding refractive index of diluted glycerol increases. The values of $\lambda$ LSPR when the glycerol samples were in contact with the nanopillars were averaged and plotted as a function of the RI variations $\left(\Delta \lambda_{\text {LSPR }}\right.$ VS. RI) displaying a linear dependence, as shown in Fig. 4c. By fitting to a linear regression function, we determined the bulk sensitivity $\left(S_{B}\right)$ values for each biosensor configuration. As a result, the effective sensitivity of the $310 \mathrm{M}$ sensor was $206 \mathrm{~nm} / \mathrm{RIU}$, and $164 \mathrm{~nm} / \mathrm{RIU}$ for the OG142 sensor (Table 1). The sensitivity of the $310 \mathrm{M}$ sensor was slightly higher than the OG142 one due to the size and mechanical properties of the polymer nanostructures. The height and flexibility of the nanopillars can help to better expose the LSPR of the Au nanodisks to the dielectric environment, improving the access of the glycerol molecules, thereby influencing the sensitivity. The obtained sensitivity values in bulk agreed with reported values ${ }^{20,34-36}$ accrediting the feasibility of our devices for sensing applications.
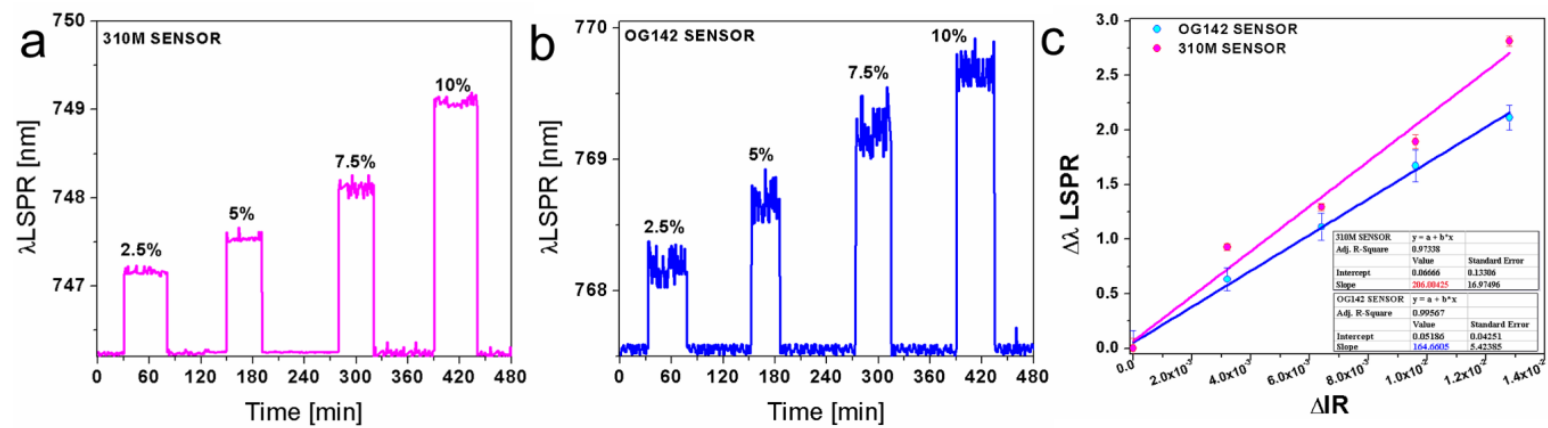

Fig. 4 Time-resolved response caused by changes in the refractive index of the surrounding dielectric medium measured by transmission for $310 \mathrm{M}$ (a) and OG142 (b) sensors. (c) Data plotted as a function of the RI variations ( $\Delta \mathrm{n} \approx 0.0032$ RIU) showing a linear dependence.

Table 1 Comparison of the bulk sensitivity

\begin{tabular}{c|c|c|c|c|c}
\hline $\left.\mathrm{d}_{\mathrm{cc}}{ }^{\mathrm{a}}\right)=400 \mathrm{~nm}$ & $\mathrm{~d}_{\mathrm{pnp}}{ }^{\mathrm{b})}(\mathrm{nm})$ & $\mathrm{h}^{\mathrm{c})}(\mathrm{nm})$ & $\begin{array}{c}\text { Aspect } \\
\text { ratio }\end{array}$ & $\begin{array}{c}(\mathrm{S}) \\
(\mathrm{nm} / \mathrm{RIU})\end{array}$ & Material \\
\hline
\end{tabular}




\begin{tabular}{l|c|c|c|c|l}
\hline $310 \mathrm{M}$ biosensor & $160 \pm 10.8$ & $320 \pm 115.1$ & 2.09 & 206 & $310 \mathrm{M}(4.16 \mathrm{MPa})$ \\
\hline OG142-87 biosensor & $185.8 \pm 18.0$ & $490 \pm 131.6$ & 2.63 & 164 & OG142-87 (3.59 GPa) \\
\hline Example 1 & 100 & 20 & 5 & 222 & Glass $^{35}$ \\
\hline Example 2 & 230 & 150 & 1.5 & 375 & $\begin{array}{l}\text { Polymethyl methacrylate } \\
34\end{array}$ \\
\hline
\end{tabular}

a) $\left(d_{\text {pnp }}\right.$ diameter of polymer nanopillar); b) $\left(d_{c c}\right.$ center to center distance); ${ }^{c}$ height

\section{Scanning Electron Microscopy characterization}

To evaluate if the differential surfaces of the biosensors were suitable for cell-adhesion, a cell assay was performed. For this, fibroblasts were seeded for 3 hours on the biosensors. Fig. 5 exhibits the adherent fibroblasts, which formed dynamic extensions during the process of cellular adhesion and spreading on the $310 \mathrm{M}$ and OG142 nanopillars. Interestingly, the fibroblasts were able to bend the nanopillars of both devices causing a shear stress with their filopodia projections similar to other reported cellular studies. ${ }^{15,37-39}$ As a result, the gold plasmonic disks approached one another.

The SEM images displayed fibroblasts with filopodial extensions of $\approx 12.49 \mu \mathrm{m}$ on the $310 \mathrm{M}$ biosensor and of $\approx 32.23 \mu \mathrm{m}$ on the OG142 biosensor. We also noticed that the Au-capped polymer nanopillars were bent by capillary forces during the liquid drying step required for fixing the attached fibroblasts for SEM characterization (Fig. 5a). Importantly, this drying step was excluded while acquiring the LSPR spectra of live fibroblasts in our biosensors and therefore not interfering in cell-nanopillar interactions.

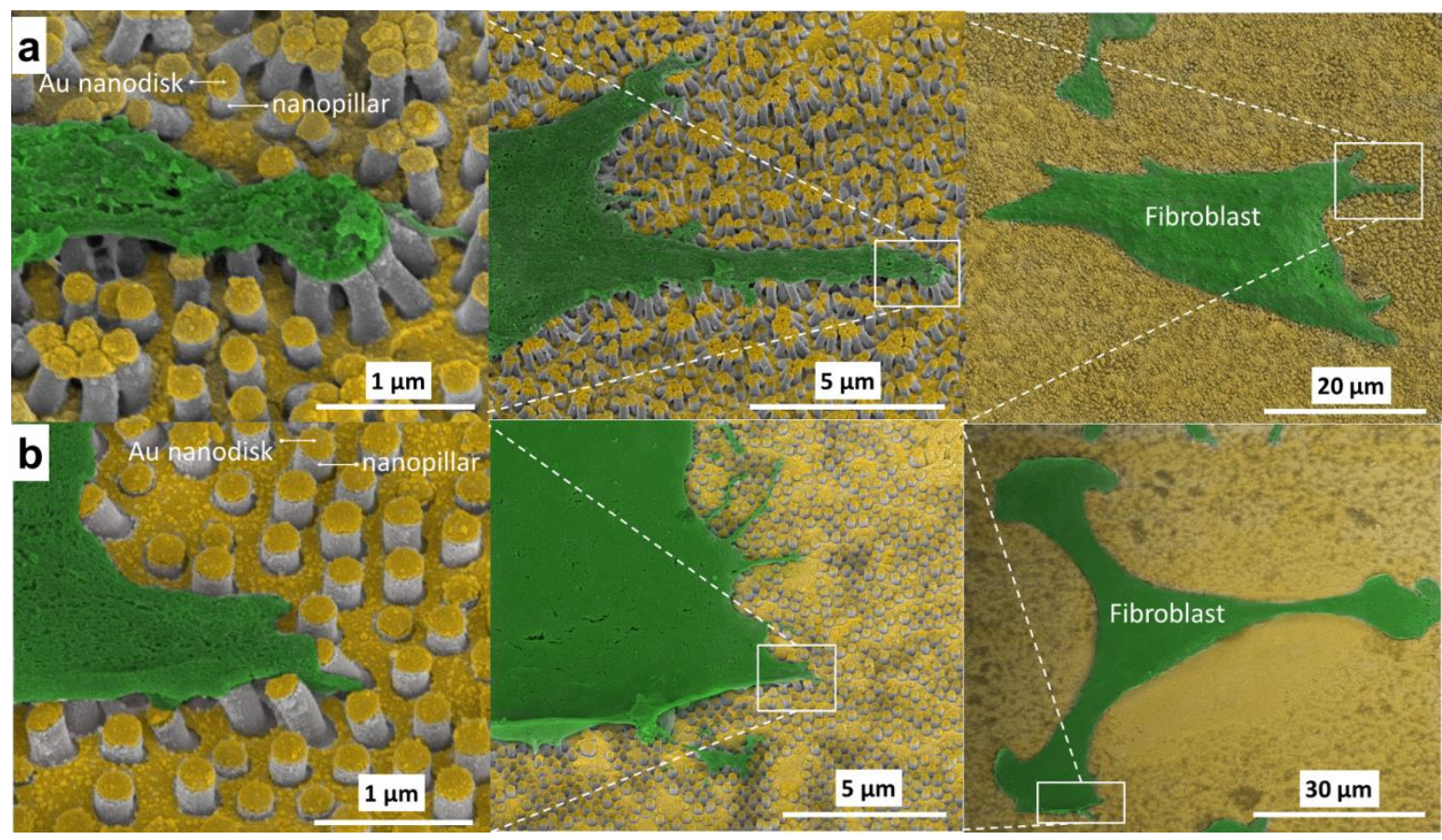

Fig. 5 Cell-nanopillar interactions and focal adhesion formation. Adherent fibroblasts (green) forming filopodial extensions during the process of cellular adhesion and spreading on (a) $310 \mathrm{M}$ and (b) OG142 nanopillars with gold nanodisks (yellow), respectively. 


\section{LSPR response of the basal media}

We verified whether the DMEM components or cell respiration could affect the LSPR spectra measurements or not. Fig. 6 shows the LSPR spectra response due to changes in the refractive index $\left(\Delta \lambda_{\mathrm{LSPR}}\right)$ of the basal media (DMEM solution, pink diamond). We noticed that these changes in the refractive index were stable over time showing a random variation of less than $\approx 0.5 \mathrm{~nm}$. DMEM solution maintained its properties and conditions due to the microfluidic channel high-quality sealing and it did not produce significant changes in the refractive index for three hours.

Also, comparing the basal media alone with the basal media pre-incubated (supernatant) with the fibroblasts (pink diamond with line, see Fig. 6), we noticed that the supernatant without fibroblasts induced changes in the LSPR producing a wavelength red-shift of less than $2 \mathrm{~nm}$ in 3 hours or $0.5 \mathrm{~nm}$ in half an hour. These subtle variations in the LSPR spectra might be attributed to cell respiration (consumed oxygen $\left(\mathrm{O}_{2}\right)$ and produced carbon dioxide $\left(\mathrm{CO}_{2}\right)$ of live fibroblasts and to the presence of other molecules, see Fig. 6 .

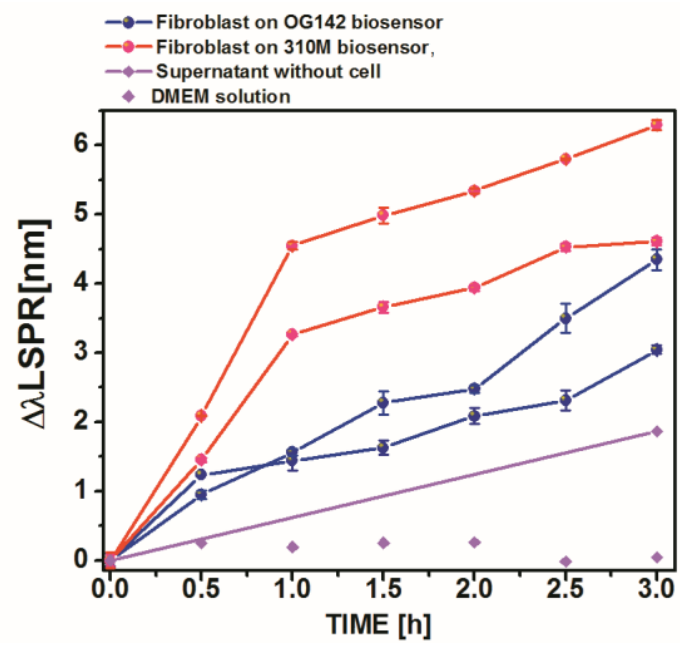

Fig. 6 Experimental $\Delta \lambda$ LSPR transmission spectra behaviours observed from fibroblasts insertion to the microfluidic channel from 0 to $3 \mathrm{~h}$ on $310 \mathrm{M}$ and OG142 biosensors. The graph shows the $\Delta \lambda L S P R$ calculated from the induced spectral resonance shift with respect to the initial position of the LSPR at $0 \mathrm{~h}$ for both biosensors. The plot also shows the LSPR responses of basal media pre-incubated with the fibroblasts (supernatant) and the LSPR corresponding to basal media alone. The slight variations of the LSPR values are attributed to electronic noise of the optical system, and signal processing of the spectra. 


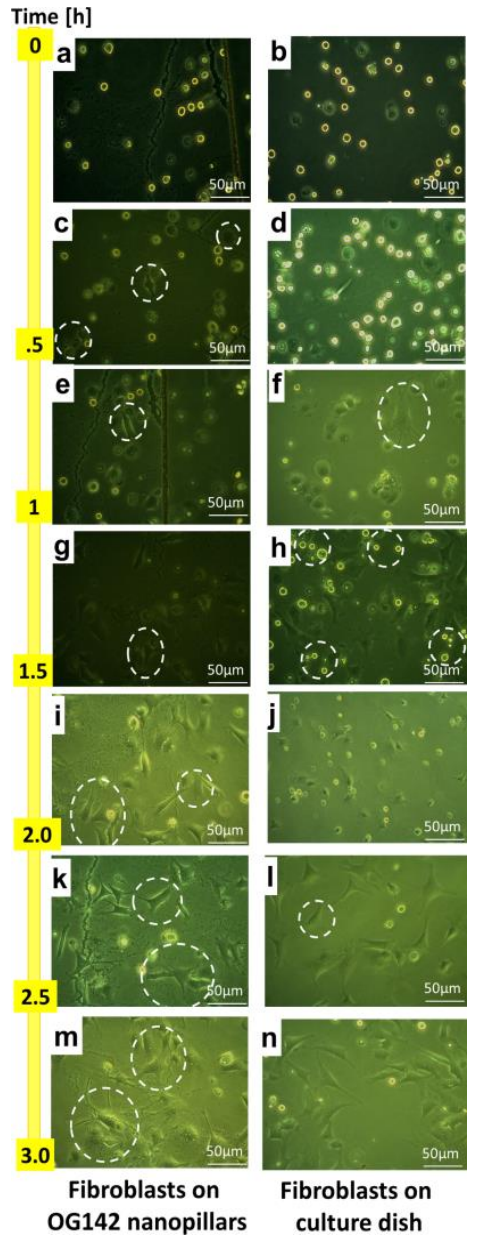

Fig. 7 Comparison of cell adhesion on OG142-87 nanopillars against the conventional plate. Optical microscopy images of fibroblasts cultured on the Au-capped OG142-87 nanopillars within the microchannel and on the conventional culture plate. Cell adherence was monitored for 3 hours; images were acquired every 30 minutes.

Since the analysis of the culture media of the fibroblasts over time showed minor contribution on the LSPR changes, we verified the plasmo-nanomechanical feature of our biosensors in order to detect LSPR changes due to the adhesion and spreading of live fibroblasts over the polymer nanopillars. Inspired by the experimental deflections of the polymer nanopillars observed by SEM imaging of the fibroblasts, we performed FDTD simulations in order to analyze the LSPR shift induced by the deflection of Au-capped polymer nanopillars (see Fig. S7†). Simulation data showed a linear relationship between the LSPR shift and the displacement of the gold nanodisks by the deflection of the nanopillars (for more details see supporting information ${ }^{\dagger}$ ).

To experimentally demonstrate this phenomenon, we injected fibroblasts into the microfluidic channel targeting the different biosensors and measured the transmission spectra for $3.0 \mathrm{~h}$. Fig. 6 shows the results of the LSPR response due to cell seeding on 310M and OG142 biosensors.

The value of $\triangle \lambda L S P R$ at $0.0 \mathrm{~h}$ corresponds to the basal media assuming that the polymer nanopillars were vertically positioned. Optical microscopy images at 20 amplifications in Fig. 7 show the comparisons between the pleating on a conventional cell-culture plate and OG142 nanopillars. Both surfaces show mainly spherical fibroblasts floating in the basal media while a few of them began to adhere to the base substrates (Figs. 7a and b). 
After $0.5 \mathrm{~h}$, fibroblasts tethering along with filopodia exploration became evident, and seemed to begin faster on arrays of nanopillars compared to the conventional cell culture dish. See Figs. 7c and d (shown in dashed circle). Fig. 6 shows that the $\Delta \lambda L S P R$ red shift caused by the cell-nanopillars interactions in average $1.3 \mathrm{~nm}$ greater than that of the basal media.

After $1.0 \mathrm{~h}$ of injection, the LSPR responses of 310M and OG142 biosensors indicated a red shift of $3.5 \mathrm{~nm}$ and $1.4 \mathrm{~nm}$ in average, respectively. For this time, the microscope images (Figs. 7e and f) show that the adherence of fibroblasts on the nanopillars was similar to that observed on the conventional dish. It is important highlight, that the increase in cell attachment on both biosensors caused a LSPR shift in average $2.5 \mathrm{~nm}$ greater than that of basal media, as shown in Fig. 6 .

The LSPR responses of the biosensors at $1.5 \mathrm{~h}$ (Fig.6) show a red shift of $4 \mathrm{~nm}$ and $2 \mathrm{~nm}$ in average, respectively. Since these increments were already greater than those of the basal media after $3 \mathrm{~h}$ (by $1.5 \mathrm{~nm}$ in average), we deduced that the greatest contribution to $\Delta \lambda$ LSPR changes was caused by live fibroblast-nanopillar interactions. Furthermore, some spherical fibroblasts were still not adhered to the media in the conventional dish (Fig $7 \mathrm{~h}$, shown in dashed circle). Conversely, most fibroblasts had adhered to the nanopillars by then (Fig $7 \mathrm{~g}$ ).

Microscopy images from $2.0 \mathrm{~h}$ to $3.0 \mathrm{~h}$ (Figs. 7i-n) clearly show that most of the adherent fibroblasts respond to the nanoscale topography via the cytoskeletal projections (filopodia) in order to explore the nanofabricated substrate. This behaviour is consistent with previously reported experiments in the literature. ${ }^{38,40-42}$ The LSPR responses of the biosensors were significantly larger (by $4.5 \mathrm{~nm}$ in average) compared to the basal media quantifications for the times between 2.0 and $3.0 \mathrm{~h}$, see Fig. 6 .

The previous results showed that live fibroblasts were able to adhere and exert shear stress at the tip of flexible nanopillars causing displacements in the Au nanodisks. Such displacements were evidenced by the $\Delta \lambda L S P R$ response quantifications. The microscopy images confirmed the biocompatibility of the $310 \mathrm{M}$ and OG142-87 materials. Finally, we analyzed that the significant $\Delta \lambda$ LSPR changes detected by the biosensors, were due to the adherence behavior, tethering and spreading of the fibroblasts, as well as a slight contribution of the culture media.

\section{Estimated adhesion forces and LSPR response}

We have employed equation (1.1) to calculate the exerted forces (e.g. cellular forces) onto single polymer nanopillars to compare our results with previous approaches. ${ }^{15,30,39}$ The dimensions and spring constants of the polymer nanopillars used for calculations are included in Table 1 and Table S1†, respectively. As follows, the displacement of the nanopillars $(\Delta \mathrm{x})$ was estimated using SEM images (Fig. 5), and the image processing software (ImageJ). The forces were calculated after $3 \mathrm{~h}^{26}$ of seeding the fibroblasts onto the gold-capped polymer nanopillars to guarantee cell adhesion and confluence. The results can be seen in Fig. 8 . 

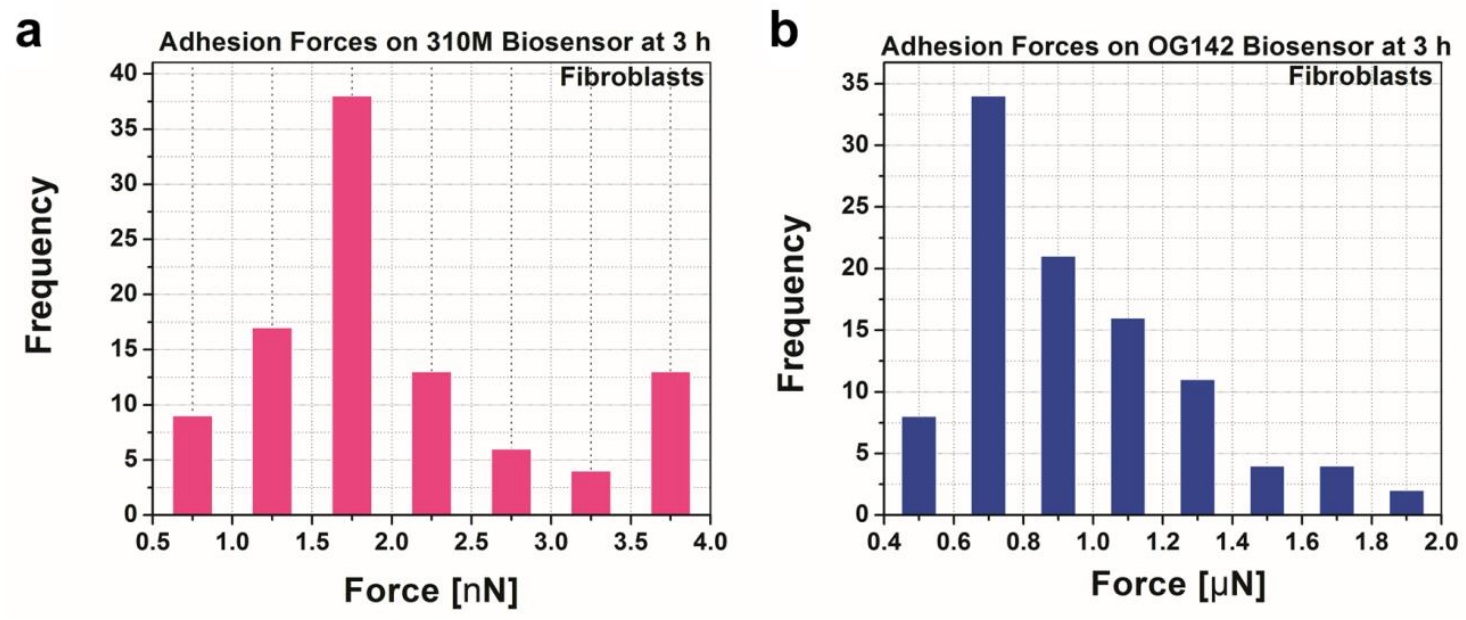

Fig. 8. Histograms of force distribution exerted at 3 hours by fibroblasts on the biosensors with different spring constant. The forces detected for the polymer nanopillars (100 counts) underlying the fibroblasts (see Fig. 5) were collected into histograms. a) Distribution of the adhesion forces on the $310 \mathrm{M}$ biosensor with a spring constant of $1.19 \mathrm{E}-02[\mathrm{~N} / \mathrm{m}]$. b) Distribution of the adhesion forces on the OG142 biosensor with a spring constant of $5.35 \mathrm{E}+00$ $[\mathrm{N} / \mathrm{m}]$.

We noticed some data dispersion in the magnitude of forces that we attribute to the mechanical interaction and adaptation of the fibroblasts to the polymer nanopillars (see Fig. $8 \mathrm{a}$ and $8 \mathrm{~b}$ ). Based on our calculations, the fibroblasts exerted on 310M and OG142 nanopillars an average force of $1.98 \pm .86 \mathrm{nN}$ and $.942 \pm .32 \mu \mathrm{N}$, respectively. We compared our estimated adhesion forces with the ones reported in the literature in order to validate our results (see Table S2+). We observed that the range of estimated forces of our biosensors $(n N-\mu N)$ was in accordance with other approaches based on structured arrays, even when those used other quantification methods. It is important to highlight the complexity of such comparison because each sensor device has been designed to measure a specific range of forces (from $\mathrm{pN}$ to $\mu \mathrm{N}$ ), and fabricated with different materials or micro/nano structure topology. The methodology of quantification of forces can also be distinct, and therefore values of the cellular responses may vary.

Since we have acquired an average response of the LSPR using our experimental setup, we can only provide an average exerted force by the fibroblasts under a surface area of ca. $7500 \mu \mathrm{m}^{2}$ (surface determined by the optical aperture of the optical setup). Based on the above data, the interplay relationship found between the LSPR shift of gold capped nanopillars and fibroblast adhesion forces was: $\overline{\Delta \lambda L S P R}_{310 M}=5.44 \pm 0.25 \mathrm{~nm}, \bar{F}_{310 M}=1.98 \pm 0.86 \mathrm{nN}$, and $\overline{\Delta \lambda L S P R}_{O G 142}=3.69 \pm 0.25$ $\mathrm{nm}, \bar{F}_{O G 142}=0.942 \pm 0.32 \mu \mathrm{N}$. We assumed that fibroblasts were able to adapt to the base substrate to imitate its properties, as is the case of rigidness. Thus, the applied force that fibroblast exerted at the tip of nanopillars was directly proportional to the rigidity of the substrate $\left(E \approx F_{\text {exerted }}\right)$ as shown in another microscale approach ${ }^{43}$. This explains the order of magnitude difference ( $\mu \mathrm{N}-\mathrm{nN}$ ) between the resulting forces of soft (310M) and rigid (OG120) nanopillars. In relation to the $\Delta \lambda L S P R$, there is an inversely proportional relationship between the calculated forces with respect to the LSPR shift and to the displacement of the gold nanodisks. For instance, rigid nanopillars (OG142) exhibited short displacements of the gold-capped nanopillars, and thus a short LSPR shift, but greater calculated forces.

In this rough force estimation based on LSPR, we ignored locally differences in stiffness of the nanopillars due to inconsistencies from the fabrication method. Likewise, the effects of bending of the nanopillars because of low flow rates for cell seeding were negligible. It should be noted that our analysis could not differentiate cell contractile forces applied to individual nanopillars to measure a local distribution of cell coupling forces to nanopillars. To achieve more accurate measurements of adherent forces with our present approach, we would require a more complex system to acquire the LSPR in real-time.

\section{Conclusions}


We have described a controlled methodology to fabricate a novel plasmo-nanomechanical biosensor within a microfluidic channel for studying cell contractile forces. By combining our fabrication method with the mechanical properties (e.g., viscosity and Young's Modulus) of EPOTECK-OG142-87 and 310M polymers, we were able to modify the substrate rigidity along with its nanoscale topography. The use of these polymers allowed us to fabricate vertically aligned nanopillars tuning their spring constants $(k)$ in order to mimic soft and rigid tissues and to control the mechanical sensitivity to shear stress. In relation to the plasmonic behavior of our sensors, the $310 \mathrm{M}$ sensor showed a bulk sensitivity of $\approx 204 \mathrm{~nm} / \mathrm{RIU}$ whereas the OG142 biosensor showed a sensitivity of $\approx 164 \mathrm{~nm} /$ RIU. SEM images showed that fibroblasts responded to the nanoscale topography of the sensors exhibiting cytoskeletal projections (filopodia), and that they exerted shear forces at the tip of flexible nanopillars causing the displacement of the gold nanodisks. We found an inversely proportional relationship between the contractile fibroblast forces with respect to the LSPR shift and to the displacement of the gold nanodisks. Based on our calculations, there was an order of magnitude difference $(\mu \mathrm{N}-\mathrm{nN})$ between the calculated average forces of soft (310M) and rigid (OG142) nanopillars. We assumed that fibroblasts were able to adapt to the base substrate to imitate its properties, as is the case of rigidness. We conclude that our approach represents a novel tool that could be employed for studying the attachment process of living cells.

\section{Author's contributions}

V. Solis-Tinoco was responsible for the nanofabrication and characterization of the sensors. Performed the optical simulations, cellular experiments on the sensors and SEM characterizations. Prepared the published work, specifically wrote the initial draft. S. Marquez contributed in the design of the bonding process of the sensors, literature review and writing. T. Quesada-Lopez and F. Villarroya contributed in the realization of the cellular experiments on the sensors. Provided the reagents, fibroblasts cells, the Biology facilities and equipment. A. Homs-Corbera contributed to the design of the optical simulations. Designed the cellular experiments on the sensors. L. M. Lechuga devised, directed and supervised the complete project. Provided all materials and the clean room facilities to develop the sensors. All authors discussed the results and approved the final manuscript.

\section{Conflicts of interest}

There are no conflicts to declare.

\section{Acknowledgements}

V. Solis-Tinoco, S. Marquez, and T. Quesada-López acknowledge financial support from "Programa becas en el extranjero" from National Council for Science and Technology (CONACYT-Mexico). The ICN2 is funded by the CERCA programme/ Generalitat de Catalunya. The ICN2 is supported by the Severo Ochoa programme of the Spanish Ministry of Science, Innovation and Universities (Grant No. SEV-2017-0706).

\section{References}

1. Ladoux B, Nicolas A. Physically based principles of cell adhesion mechanosensitivity in tissues. Rep Prog Phys. 2012;75(11):116601.

2. Thiery JP. Cell adhesion in cancer. Comptes Rendus Phys. 2003 Mar 1;4(2):289-304.

3. Yang Y, Wang K, Gu X, Leong KW. Biophysical Regulation of Cell Behavior-Cross Talk between Substrate Stiffness and Nanotopography. Engineering. 2017 Feb 1;3(1):36-54.

4. Ahmad Khalili A, Ahmad MR. A Review of Cell Adhesion Studies for Biomedical and Biological Applications. Int J Mol Sci. 2015 Aug 5;16(8):18149-84.

5. Thompson MT, Berg MC, Tobias IS, Lichter JA, Rubner MF, Van Vliet KJ. Biochemical Functionalization of Polymeric Cell Substrata Can Alter Mechanical Compliance. Biomacromolecules. 2006 Jun 1;7(6):1990-5. 
6. Tallawi M, Rosellini E, Barbani N, Cascone MG, Rai R, Saint-Pierre G, et al. Strategies for the chemical and biological functionalization of scaffolds for cardiac tissue engineering: a review. J R Soc Interface. 2015 Jul 6;12(108):20150254.

7. Guo W, Frey MT, Burnham NA, Wang Y. Substrate rigidity regulates the formation and maintenance of tissues. Biophys J. 2006 Mar 15;90(6):2213-20.

8. Rowlands AS, George PA, Cooper-White JJ. Directing osteogenic and myogenic differentiation of MSCs: interplay of stiffness and adhesive ligand presentation. Am J Physiol Cell Physiol. 2008 Oct;295(4):C1037-1044.

9. Engler AJ, Sen S, Sweeney HL, Discher DE. Matrix elasticity directs stem cell lineage specification. Cell. 2006 Aug $25 ; 126(4): 677-89$.

10. al GK et. Cell adaptation to a physiologically relevant ECM mimic with different viscoelastic properties. - PubMed NCBI.

11. Liu WF. Mechanical regulation of cellular phenotype: implications for vascular tissue regeneration. Cardiovasc Res. 2012 Jul 15;95(2):215-22.

12. Saez A, Ghibaudo M, Buguin A, Silberzan P, Ladoux B. Rigidity-driven growth and migration of epithelial cells on microstructured anisotropic substrates. Proc Natl Acad Sci. 2007 May 15;104(20):8281-6.

13. Roure $\mathrm{O} d u$, Saez A, Buguin A, Austin RH, Chavrier $P$, Siberzan $P$, et al. Force mapping in epithelial cell migration. Proc Natl Acad Sci U S A. 2005 Feb 15;102(7):2390-5.

14. Fu J, Wang Y-K, Yang MT, Desai RA, Yu X, Liu Z, et al. Mechanical regulation of cell function with geometrically modulated elastomeric substrates. Nat Methods. 2010 Sep;7(9):733-6.

15. M.T Y, N.J S, C.S C. Geometric Considerations of Micro- to Nanoscale Elastomeric Post Arrays to Study Cellular Traction Forces. Adv Mater. 2007;3119-23.

16. Tan JL, Liu W, Nelson CM, Raghavan S, Chen CS. Simple approach to micropattern cells on common culture substrates by tuning substrate wettability. Tissue Eng. 2004 Jun;10(5-6):865-72.

17. Hong S, Ergezen E, Lec R, Barbee KA. Real-time analysis of cell-surface adhesive interactions using thickness shear mode resonator. Biomaterials. 2006 Dec;27(34):5813-20.

18. Wang W, Wang S, Liu Q, Wu J, Tao N. Mapping Single-Cell-Substrate Interactions by Surface Plasmon Resonance Microscopy. Langmuir. 2012 Sep 18;28(37):13373-9.

19. Soler M, Estevez M-C, Moreno M de L, Cebolla A, Lechuga LM. Label-free SPR detection of gluten peptides in urine for non-invasive celiac disease follow-up. Biosens Bioelectron. 2016 May 15;79:158-64.

20. Soler M, Estevez M-C, Villar-Vazquez R, Casal JI, Lechuga LM. Label-free nanoplasmonic sensing of tumor-associate autoantibodies for early diagnosis of colorectal cancer. Anal Chim Acta. 2016 Aug 3;930:31-8.

21. Yang C-T, Méjard R, Griesser HJ, Bagnaninchi PO, Thierry B. Cellular micromotion monitored by long-range surface plasmon resonance with optical fluctuation analysis. Anal Chem. 2015 Feb 3;87(3):1456-61.

22. Solis-Tinoco V, Marquez S, Sepulveda B, Lechuga LM. Fabrication of well-ordered silicon nanopillars embedded in a microchannel via metal-assisted chemical etching: a route towards an opto-mechanical biosensor. RSC Adv. 2016 Sep 6;6(88):85666-74.

23. Solis-Tinoco V, Sepulveda B, Lechuga LM. Novel nanoplasmonic biosensor integrated in a microfluidic channel. In Barcelona,Spain; 2015. p. 95190T-95190T-9. 
24. Eddings MA, Johnson MA, Gale BK. Determining the optimal PDMS-PDMS bonding technique for microfluidic devices. J Micromechanics Microengineering. 2008 Jun 1;18(6):067001.

25. Bhattacharya S, Datta A, Berg JM, Gangopadhyay S. Studies on surface wettability of poly(dimethyl) siloxane (PDMS) and glass under oxygen-plasma treatment and correlation with bond strength. J Microelectromechanical Syst. 2005 Jun;14(3):590-7.

26. Yang MT, Fu J, Wang Y-K, Desai RA, Chen CS. Assaying stem cell mechanobiology on microfabricated elastomeric substrates with geometrically modulated rigidity. Nat Protoc. 2011 Jan;6(2):187-213.

27. Harlow E, Lane D. Fixing Attached Cells in Paraformaldehyde. Cold Spring Harb Protoc. 2006 Jan 8;2006(3):pdb.prot4294.

28. Hui CY, Jagota A, Lin YY, Kramer EJ. Constraints on Microcontact Printing Imposed by Stamp Deformation. Langmuir. 2002 Feb 1;18(4):1394-407.

29. Sharp KG, Blackman GS, Glassmaker NJ, Jagota A, Hui C-Y. Effect of stamp deformation on the quality of microcontact printing: theory and experiment. Langmuir ACS J Surf Colloids. 2004 Jul 20;20(15):6430-8.

30. Ghibaudo M, Saez A, Trichet L, Xayaphoummine A, Browaeys J, Silberzan P, et al. Traction forces and rigidity sensing regulate cell functions. Soft Matter. 2008 Aug 12;4(9):1836-43.

31. Delanoë-Ayari H. 4D Traction Force Microscopy Reveals Asymmetric Cortical Forces in Migrating Dictyostelium Cells. Phys Rev Lett. 2010;105(24).

32. Maruthamuthu V, Sabass B, Schwarz US, Gardel ML. Cell-ECM traction force modulates endogenous tension at cellcell contacts. Proc Natl Acad Sci. 2011 Mar 22;108(12):4708-13.

33. Trepat X, Wasserman MR, Angelini TE, Millet E, Weitz DA, Butler JP, et al. Physical forces during collective cell migration. Nat Phys. 2009 Jun;5(6):426-30.

34. Yang S-C, Hou J-L, Finn A, Kumar A, Ge Y, Fischer W-J. Synthesis of multifunctional plasmonic nanopillar array using soft thermal nanoimprint lithography for highly sensitive refractive index sensing. Nanoscale. 2015 Mar 19;7(13):5760-6.

35. Otte MA, Estévez M-C, Carrascosa LG, González-Guerrero AB, Lechuga LM, Sepúlveda B. Improved Biosensing Capability with Novel Suspended Nanodisks. J Phys Chem C. 2011 Apr 7;115(13):5344-51.

36. Chung T, Lee S-Y, Song EY, Chun H, Lee B. Plasmonic Nanostructures for Nano-Scale Bio-Sensing. Sensors. 2011 Nov 21;11(11):10907-29.

37. Tan JL, Tien J, Pirone DM, Gray DS, Bhadriraju K, Chen CS. Cells lying on a bed of microneedles: An approach to isolate mechanical force. Proc Natl Acad Sci. 2003 Feb 18;100(4):1484-9.

38. Trichet L, Le Digabel J, Hawkins RJ, Vedula SRK, Gupta M, Ribrault C, et al. Evidence of a large-scale mechanosensing mechanism for cellular adaptation to substrate stiffness. Proc Natl Acad Sci U S A. 2012 May 1;109(18):6933-8.

39. Digabel J le, Ghibaudo M, Trichet L, Richert A, Ladoux B. Microfabricated substrates as a tool to study cell mechanotransduction. Med Biol Eng Comput. 2010 Oct 1;48(10):965-76.

40. Balaban NQ, Schwarz US, Riveline D, Goichberg P, Tzur G, Sabanay I, et al. Force and focal adhesion assembly: a close relationship studied using elastic micropatterned substrates. Nat Cell Biol. 2001 May;3(5):466-72.

41. Lo C-M, Wang H-B, Dembo M, Wang Y. Cell Movement Is Guided by the Rigidity of the Substrate. Biophys J. 2000 Jul;79(1):144-52.

42. Dalby MJ. Cellular response to low adhesion nanotopographies. Int J Nanomedicine. 2007 Sep;2(3):373-81. 
43. Tanaka Y, Morishima K, Shimizu T, Kikuchi A, Yamato M, Okano T, et al. Demonstration of a PDMS-based biomicroactuator using cultured cardiomyocytes to drive polymer micropillars. Lab Chip. 2006 Feb;6(2):230-5. 